PROCEEDINGS OF THE

AMERICAN MATHEMATICAL SOCIETY

Volume 134, Number 8, Pages 2259-2270

S 0002-9939(06)08239-6

Article electronically published on January 31, 2006

\title{
TAYLOR SERIES FOR THE ASKEY-WILSON OPERATOR AND CLASSICAL SUMMATION FORMULAS
}

\author{
BERNARDO LÓPEZ, JOSÉ MANUEL MARCO, AND JAVIER PARCET \\ (Communicated by Christopher D. Sogge)
}

\begin{abstract}
An analogue of Taylor's formula, which arises by substituting the classical derivative by a divided difference operator of Askey-Wilson type, is developed here. We study the convergence of the associated Taylor series. Our results complement a recent work by Ismail and Stanton. Quite surprisingly, in some cases the Taylor polynomials converge to a function which differs from the original one. We provide explicit expressions for the integral remainder. As an application, we obtain some summation formulas for basic hypergeometric series. As far as we know, one of them is new. We conclude by studying the different forms of the binomial theorem in this context.
\end{abstract}

\section{INTRODUCTION AND DEFINITIONS}

The problem of expanding a function with respect to a given polynomial basis has many implications in analysis. The simplest example of this kind is Taylor's expansion theorem. In this paper, we replace the classical derivative by a difference operator of Askey-Wilson type. Our results complement the paper [3] of Ismail and Stanton and are a natural continuation of the point of view presented in [5], where a new approach to the theory of classical hypergeometric polynomials is given. In contrast with 3, our aim is to find sufficient conditions for the Taylor series to converge, but not necessarily to the original function. In this more general setting, we may consider non-necessarily entire functions and we give an explicit expression for the limit of the remainders in terms of a contour integral. Using this and a new estimate for the $q$-shifted factorials, which might be of independent interest, we obtain a summation formula which is new as far as we know. As we explain below, it can be regarded as a non-symmetrized version of the non-terminating $q$-Saalschütz sum. As applications, we also provide a new proof of the $q$-Gauss summation formula and a list of binomial type summation formulas in the same line as Ismail's paper [2].

Now we give some definitions which will be used in what follows. The notions we are presenting were already introduced in $[5]$ with the aim of studying some aspects of the theory of hypergeometric polynomials. The relevance of this approach is justified in [5], where a more detailed exposition is given.

Received by the editors May 17, 2004 and, in revised form, February 24, 2005.

2000 Mathematics Subject Classification. Primary 33D15.

Key words and phrases. q-Taylor series, Askey-Wilson operator, basic hypergeometric function.

(C)2006 American Mathematical Society Reverts to public domain 28 years from publication 
Definition 1.1. Let $\mathcal{P}$ be the set of quadratic symmetric polynomials $\mathrm{P}(x, y)$, normalized so that $\mathrm{P}(x, y)=x^{2}+y^{2}-2 a x y-2 b(x+y)+c$ with $a, b, c$ complex numbers. A sequence of complex numbers $\left(x_{t}\right)$, with the index $t$ running over the set $\frac{1}{2} \mathbb{Z}=\{k / 2: k \in \mathbb{Z}\}$, will be called a $\mathrm{P}$-sequence if

$$
\mathrm{P}\left(x_{t}, y\right)=\left(y-x_{t+\frac{1}{2}}\right)\left(y-x_{t-\frac{1}{2}}\right) \quad \text { for all } t \in \frac{1}{2} \mathbb{Z} .
$$

Recalling the natural action of the affine group of the complex plane $\operatorname{Aff}(\mathbb{C})$ on the set $\mathcal{P}$, we can consider the orbits of this action. As it becomes clear in [5], these orbits provide a very natural classification in the theory. In particular, each symmetric polynomial in $\mathcal{P}$ can be rewritten, under affine transformations, in a canonical form. In Table I, we summarize the canonical forms and the corresponding $\mathrm{P}$-sequences which we shall use in our study. We shall also use the parameter $\lambda \neq 0$ defined by the relation $a=\frac{1}{2}\left(\lambda+\lambda^{-1}\right)$. Here we recall that the basis $q$ will be given by $\lambda^{2}$.

TABLE I. Canonical P-sequences

\begin{tabular}{l}
\multicolumn{2}{c}{ Canonical form } & P-sequence \\
\begin{tabular}{|c|c|c|}
\hline $\mathbf{T}$ & $x^{2}+y^{2}-2 a x y+a^{2}-1$ & $\frac{1}{2}\left(\lambda^{2 t} u+\lambda^{-2 t} u^{-1}\right)$ \\
\hline $\mathbf{G}$ & $x^{2}+y^{2}-2 a x y$ & $\lambda^{2 t} u$ \\
\hline $\mathbf{Q}$ & $(x-y)^{2}-\frac{1}{2}(x+y)+\frac{1}{16}$ & $t^{2}+2 t u+u^{2}$ \\
\hline $\mathbf{A}$ & $(x-y)^{2}-\frac{1}{4}$ & $t+u$ \\
\hline $\mathbf{C}$ & $(x-y)^{2}$ & $u$ \\
\hline
\end{tabular}
\end{tabular}

In the first two rows we assume $a \neq \pm 1$. The capital letters in the left column are acronyms of the names we adopted in 5 for the canonical forms: Trigonometric, Geometric, Quadratic, Arithmetic and Continuous. We shall say that $x_{0}$ is the base point of the P-sequence $\left(x_{t}\right)$. Obviously, $\mathrm{P}(x, y)=y^{2}-2 A(x) y+B(x)$, where $A(x)=a x+b$ and $B(x)=x^{2}-2 b x+c$. This allows us to consider the discriminant of $\mathrm{P}$ as a function of the variable $x$ which, up to a constant factor, is given by

$$
\delta(x)=\left(a^{2}-1\right) x^{2}+2 b(a+1) x+b^{2}-c .
$$

Remark 1.2. P-sequences arise from the recurrence $x_{t \pm \frac{1}{2}}=A\left(x_{t}\right) \pm \sqrt{\delta\left(x_{t}\right)}$. In particular, given a complex number $\xi$, there are at most two $\mathrm{P}$-sequences with base point $\xi$, one for each choice of the sign for the square root $\sqrt{\delta(\xi)}$.

Definition 1.3. For any complex number $x$, we consider a P-sequence $\left(x_{t}\right)$ with base point $x$. Then we define $\Phi_{0}(x, y)=1$ and the polynomials $\Phi_{k}(x, y)$, for any positive integer $k \geq 1$, as follows:

$$
\Phi_{k}(x, y)=\prod_{j=0}^{k-1}\left(y-x_{j-\frac{k-1}{2}}\right) .
$$

Remark 1.4. Recall that $\Phi_{k}(x, \cdot)$ does not depend on the chosen P-sequence $\left(x_{t}\right)$. 


\section{TAYLOR SERIES}

Given an open subset $\Omega$ of the complex plane, we denote the space of analytic functions in $\Omega$ by $\mathcal{H}(\Omega)$. Also, by $\gamma \simeq 0(\bmod \Omega)$ we mean that $\gamma$ is a cycle in $\Omega$ homologous to zero with respect to $\Omega$. Finally, given $z \in \Omega$, $\operatorname{Ind}(\gamma, z)$ denotes the index of $z$ with respect to $\gamma$.

Lemma 2.1. Given an open subset $\Omega$ of the complex plane, let us denote by $\Delta_{\Omega}$ the diagonal of $\Omega \times \Omega$. Then, for any $f \in \mathcal{H}(\Omega)$, the function

$$
f_{d}(u, v)=\frac{f(u)-f(v)}{u-v} \quad \text { for }(u, v) \in(\Omega \times \Omega) \backslash \Delta_{\Omega}
$$

can be continuously extended to an analytic function $f_{d}: \Omega \times \Omega \rightarrow \mathbb{C}$. Moreover, if $\gamma \simeq 0(\bmod \Omega)$ and $\operatorname{Ind}(\gamma, u)=\operatorname{Ind}(\gamma, v)=1$, then we have

$$
f_{d}(u, v)=\frac{1}{2 \pi i} \int_{\gamma} \frac{f(y)}{(y-u)(y-v)} d y
$$

Proof. It is a simple consequence of Cauchy's integral formula.

Let us consider the set $\Omega_{1}=\{x \in \mathbb{C}: A(x) \pm \sqrt{\delta(x)} \in \Omega\}$. By Lemma 2.1, given a quadratic symmetric polynomial $\mathrm{P} \in \mathcal{P}$, we define the Askey-Wilson operator $\mathrm{D}: \mathcal{H}(\Omega) \rightarrow \mathcal{H}\left(\Omega_{1}\right)$ as follows:

$$
\mathrm{D} f(x)=f_{d}(A(x)+\sqrt{\delta(x)}, A(x)-\sqrt{\delta(x)})=\frac{1}{2 \pi i} \int_{\gamma} \frac{f(y)}{\mathrm{P}(x, y)} d y,
$$

with $\gamma \simeq 0(\bmod \Omega)$ and $\operatorname{Ind}(\gamma, A(x) \pm \sqrt{\delta(x)})=1$. On the other hand, if we define recursively the sets $\Omega_{k}$ by $\Omega_{k+1}=\left\{x \in \mathbb{C}: A(x) \pm \sqrt{\delta(x)} \in \Omega_{k}\right\}$ with $\Omega_{0}=\Omega$, we may consider the iterated operators

$$
\mathrm{D}^{k}: \mathcal{H}(\Omega) \rightarrow \mathcal{H}\left(\Omega_{k}\right)
$$

Lemma 2.2. Let $\Omega$ be an open set of the complex plane and $\gamma \simeq 0(\bmod \Omega)$. Then, given $f \in \mathcal{H}(\Omega)$ and $x \in \Omega_{k}$, we have the following identity (with the obvious limits for $\lambda= \pm 1)$ :

$$
\mathrm{D}^{k} f(x)=\left(\prod_{j=0}^{k-1} \frac{\lambda^{k-j}-\lambda^{j-k}}{\lambda-\lambda^{-1}}\right) \frac{1}{2 \pi i} \int_{\gamma} \frac{f(y)}{\Phi_{k+1}(x, y)} d y \quad \text { for } k \geq 0
$$

if $x$ is the base point of a $\mathrm{P}$-sequence $\left(x_{t}\right)$ with $\operatorname{Ind}\left(\gamma, x_{j-\frac{k}{2}}\right)=1$ for $j=0,1, \ldots, k$. In particular, we shall consider the operator

$$
\partial_{k}: \mathcal{H}(\Omega) \rightarrow \mathcal{H}\left(\Omega_{k}\right) \quad \text { defined by } \quad \partial_{k} f(x)=\frac{1}{2 \pi i} \int_{\gamma} \frac{f(y)}{\Phi_{k+1}(x, y)} d y .
$$

Proof. The cases $k=0$ and $k=1$ follow from Cauchy's integral formula and (11) respectively. Therefore, the general case follows by induction from the relation

$$
\mathrm{D}\left[\frac{1}{\Phi_{k}(\cdot, y)}\right](x)=\frac{\Phi_{k}\left(x_{1 / 2}, y\right)^{-1}-\Phi_{k}\left(x_{-1 / 2}, y\right)^{-1}}{x_{1 / 2}-x_{-1 / 2}}=\frac{\lambda^{k}-\lambda^{-k}}{\lambda-\lambda^{-1}} \Phi_{k+1}(x, y)^{-1},
$$

which is not difficult to check with the aid of the identity

$$
\frac{x_{k / 2}-x_{-k / 2}}{x_{1 / 2}-x_{-1 / 2}}=\frac{\lambda^{k}-\lambda^{-k}}{\lambda-\lambda^{-1}}
$$

This identity was proved in [5] for any $\mathrm{P}$-sequence. This completes the proof. 
The following result provides the sequence of Taylor polynomials associated to a given function $f \in \mathcal{H}(\Omega)$ and the corresponding remainder term, with respect to the Askey-Wilson operator.

Theorem 2.3. Let $\Omega$ be an open subset of the complex plane and $\gamma \simeq 0(\bmod \Omega)$. Then, given an analytic function $f \in \mathcal{H}(\Omega)$ and $x \in \Omega$ with $\operatorname{Ind}(\gamma, x)=1$, we can recover $f(x)$ as

$$
f(x)=\sum_{k=0}^{n} \partial_{k} f\left(z_{k / 2}\right) \prod_{j=0}^{k-1}\left(x-z_{j}\right)+\mathcal{R}_{n} f(x),
$$

where $\mathcal{R}_{n} f(x)$ is given by

$$
\mathcal{R}_{n} f(x)=\frac{1}{2 \pi i} \int_{\gamma} \frac{f(y)}{y-x} \prod_{j=0}^{n} \frac{x-z_{j}}{y-z_{j}} d y,
$$

with $\left(z_{t}\right)$ a P-sequence such that $z_{j} \in \Omega$ and $\operatorname{Ind}\left(\gamma, z_{j}\right)=1$ for $j=0,1, \ldots, n$.

Proof. The relation below follows by induction on $n$ :

$$
\frac{1}{y-x}=\frac{\Phi_{n+1}\left(z_{n / 2}, x\right)}{(y-x) \Phi_{n+1}\left(z_{n / 2}, y\right)}+\sum_{k=0}^{n} \frac{\Phi_{k}\left(z_{(k-1) / 2}, x\right)}{\Phi_{k+1}\left(z_{k / 2}, y\right)} .
$$

Multiplying by $(2 \pi i)^{-1} f(y)$, we are done by Lemma 2.2 and Cauchy's formula.

Remark 2.4. When dealing with the continuous form $\mathbf{C}$, it turns out that the AskeyWilson operator can be regarded as the classical derivative; see [5] for the details. In particular, Theorem 2.3 reduces to the classical Taylor series

$$
f(x)=\sum_{k=0}^{n} \frac{f^{(k)}(z)}{k !}(x-z)^{k}+f_{n+1}(x)(x-z)^{n+1},
$$

where

$$
f_{n+1}(x)=\frac{1}{2 \pi i} \int_{\gamma} \frac{f(y)}{(y-z)^{n+1}(y-x)} d y .
$$

Remark 2.5. If the points $z_{j}$ are pairwise distinct for $j=0,1, \ldots, n$, we can use Lemma 2.2 to express $\partial_{k} f\left(z_{k / 2}\right)$ as a sum of residues and Theorem 2.3 gives

$$
f(x)-\mathcal{R}_{n} f(x)=\sum_{k=0}^{n}\left(\sum_{j=0}^{k} \frac{f\left(z_{j}\right)}{\prod_{i \neq j}\left(z_{j}-z_{i}\right)}\right) \prod_{j=0}^{k-1}\left(x-z_{j}\right),
$$

which is Newton's divided difference formula for the interpolation polynomial. In particular, Theorem 2.3 holds for any collection $z_{0}, z_{1}, \ldots, z_{n}$ of pairwise distinct points. However, as we shall see below, the relevance of Theorem 2.3 lies in the established connection with the Askey-Wilson operator.

Now we study the convergence of the Taylor series for P-sequences $\left(z_{t}\right)$ with $z_{0}, z_{1}, z_{2}, \ldots$ bounded. This covers the geometric canonical form for $|q|<1$ and the continuous form, whose associated P-sequences are constant. Given $r>0$, we shall denote by $\mathbb{D}_{\mathrm{r}}=\{z \in \mathbb{C}:|z|<\mathrm{r}\}$ the open disk of radius $\mathrm{r}$. As was pointed out by the referee, the result below is closely related to Wallisser's paper [6]. 
Theorem 2.6. Let $\left(z_{t}\right)$ be a $\mathrm{P}$-sequence satisfying that the subsequence $z_{0}, z_{1}, z_{2}, \ldots$ is bounded. Then, given $\mathbf{z}=\lim \sup _{k \geq 0}\left|z_{k}\right|, \delta>0$ and $f \in \mathcal{H}\left(\mathbb{D}_{\mathbf{r}+\delta}\right)$ with $\mathbf{r}>2 \mathbf{z}$, the Taylor polynomials

$$
\mathcal{S}_{n} f(x)=\sum_{k=0}^{n} \partial_{k} f\left(z_{k / 2}\right) \prod_{j=0}^{k-1}\left(x-z_{j}\right)
$$

converge uniformly to $f(x)$ as $n \rightarrow \infty$ on the disk $\mathbb{D}_{\mathbf{x}}$ for any $\mathbf{x}<\mathbf{r}-2 \mathbf{z}$.

Proof. By Theorem 2.3, it suffices to check that $\mathcal{R}_{n} f \rightarrow 0$ uniformly on the disk $\mathbb{D}_{\mathbf{x}}$. Let us take $\gamma$ to be a circumference centered at 0 with radius $\mathbf{r}>\mathbf{x}+2 \mathbf{z}$. It turns out that, if $\mathrm{M}_{\mathbf{r}}(f)$ denotes the supremum of $|f(y)|$ when $y$ runs over $\gamma$, we have

$$
\left|\mathcal{R}_{n} f(x)\right| \leq \mathbf{r} \frac{\mathrm{M}_{\mathbf{r}}(f)}{\mathbf{r}-\mathbf{x}}\left(\frac{\mathbf{x}+\mathbf{z}}{\mathbf{r}-\mathbf{z}}\right)^{n+1} \longrightarrow 0 \quad \text { as } n \rightarrow \infty .
$$

Since the given bound holds for every $x \in \mathbb{D}_{\mathbf{x}}$, the proof is complete.

The following is a convergence theorem with integral remainder. This case is applicable to the trigonometric and quadratic canonical forms as well as to the geometric canonical form with $|q|>1$. Let $\left(z_{t}\right)$ be a P-sequence satisfying $\sum_{k \geq 0} 1 /\left|z_{k}\right|<\infty$. Then, the product

$$
\mathrm{H}(x)=\prod_{j=0}^{\infty}\left(1-x / z_{j}\right)
$$

is an entire function of $x$ with zeros at $x=z_{j}$ for $j=0,1,2, \ldots$.

Theorem 2.7. Let $\left(z_{t}\right)$ be a $\mathrm{P}$-sequence satisfying that the subsequence $z_{0}, z_{1}, z_{2}, \ldots$ lies in $\mathbb{R}_{-}=\{x \in \mathbb{R}: x<0\}$ and $\sum_{k \geq 0} 1 /\left|z_{k}\right|<\infty$. Assume that $f$ is analytic in an open neighborhood of the left half-plane

$$
\Omega=\{z \in \mathbb{C}: \operatorname{Re} z \leq 0\}
$$

and is controlled by

$$
|f(z)| \leq \mathrm{C}(1+|z|)^{\mathrm{M}} \quad \text { for all } \quad z \in \Omega
$$

and some absolute constants $\mathrm{C}$ and $\mathrm{M}$. In that case the pointwise limit

$$
\mathcal{S}_{\infty} f(x)=\lim _{n \rightarrow \infty} \mathcal{S}_{n} f(x)
$$

exists when $\operatorname{Re} x<0$ and we have

$$
f(x)=\mathcal{S}_{\infty} f(x)+\mathcal{R}_{\infty} f(x)
$$

with

$$
\mathcal{R}_{\infty} f(x)=\frac{1}{2 \pi i} \int_{-i \infty}^{i \infty} \frac{f(y)}{y-x} \frac{\mathrm{H}(x)}{\mathrm{H}(y)} d y=\frac{1}{2 \pi i} \int_{-i \infty}^{i \infty} \frac{f(y)}{y-x} \prod_{j=0}^{\infty} \frac{x-z_{j}}{y-z_{j}} d y .
$$

Proof. According to Theorem 2.3 we have

$$
\mathcal{R}_{n} f(x)=\frac{1}{2 \pi i}\left(\int_{-i r}^{i r} \frac{f(y)}{y-x} \prod_{j=0}^{n} \frac{x-z_{j}}{y-z_{j}} d y+\int_{\gamma_{r}} \frac{f(y)}{y-x} \prod_{j=0}^{n} \frac{x-z_{j}}{y-z_{j}} d y\right),
$$

whenever $\operatorname{Re} x<0$ and where $r$ and $\gamma_{r}:[0, \pi] \rightarrow \mathbb{C}$ are determined by

$$
\max \left\{|x|,\left|z_{0}\right|,\left|z_{1}\right|, \ldots,\left|z_{n}\right|\right\}<r \quad \text { and } \quad \gamma_{r}(t)=i r e^{i t}(0 \leq t \leq \pi) .
$$


On the other hand, it easily follows from (2) that for any $y \in \gamma_{r}[0, \pi]$ we have

$$
\left|\frac{f(y)}{y-x} \prod_{j=0}^{n} \frac{x-z_{j}}{y-z_{j}}\right| \leq \mathrm{C}^{\prime}(1+r)^{\mathrm{M}-n-2},
$$

for some constant $\mathrm{C}^{\prime}$ depending on $x, z_{0}, z_{1}, \ldots, z_{n}$ but not on $y$. Therefore, since we may take the parameter $r$ arbitrarily large, we deduce that for any integer $n \geq \mathrm{M}$ we have

$$
\lim _{r \rightarrow \infty} \int_{\gamma_{r}} \frac{f(y)}{y-x} \prod_{j=0}^{n} \frac{x-z_{j}}{y-z_{j}} d y=0 .
$$

In other words, for $n \geq \mathrm{M}$ we have

$$
\mathcal{R}_{n} f(x)=\frac{1}{2 \pi i} \prod_{j=0}^{n}\left(1-x / z_{j}\right) \int_{-i \infty}^{i \infty} \frac{f(y)}{y-x} \prod_{j=0}^{n}\left(1-y / z_{j}\right)^{-1} d y .
$$

Then, estimating as above we find for $n \geq \mathrm{M}$,

$$
\left|\frac{f(y)}{y-x} \prod_{j=0}^{n}\left(1-y / z_{j}\right)^{-1}\right| \leq \mathrm{C}^{\prime \prime}(1+|y|)^{\mathrm{M}-1} \prod_{j=0}^{n}\left(1+\frac{|y|^{2}}{\left|z_{j}\right|^{2}}\right)^{-1 / 2} \leq \mathrm{C}^{\prime \prime \prime}(1+|y|)^{-2} .
$$

Therefore, the assertion follows from the dominated convergence theorem.

Remark 2.8. Similar arguments show that the convergence in Theorem 2.7 is uniform over the compact sets of $\{x \in \mathbb{C}: \operatorname{Re} x<0\}$. On the other hand, it is clear that Theorem 2.7 can be restated by taking $z_{0}, z_{1}, z_{2}, \ldots$ in $\mathbb{R}_{+}$,

$$
\Omega=\{z \in \mathbb{C}: \operatorname{Re} z \geq 0\} \quad \text { and } \quad \mathcal{R}_{\infty} f(x)=\frac{-1}{2 \pi i} \int_{-i \infty}^{i \infty} \frac{f(y)}{y-x} \prod_{j=0}^{\infty} \frac{x-z_{j}}{y-z_{j}} d y .
$$

This shows that the quadratic P-sequences are contemplated by Theorem 2.7. We have emphasized this point following a suggestion from the referee. However, when dealing with the trigonometric form, we might consider a growth restriction weaker than (2). We omit the proof for lack of space and refer the reader to [3].

Remark 2.9. The main result in Ismail/Stanton's paper [3, Theorem 3.1] considers Taylor series of entire functions with respect to the trigonometric canonical form. Our main contribution in Theorem 2.7 is that we do not require $f$ to be entire. Note that the regularity and growth restrictions in [3] allowed the authors to obtain the Taylor series of products of $q$-shifted factorials. Theorem 2.7 and Lemma 3.1 below allow us to consider in the next section quotients of $q$-shifted factorials.

\section{Summation formulas}

Now we compute the Taylor coefficients of a couple of functions made up of $q$-shifted factorials. Then we analyze the convergence of the corresponding Taylor series. We obtain a new proof of the $q$-Gauss summation formula and, as far as we know, a new summation formula. 
3.1. Geometric case. We begin by applying Theorem 2.6 to a particular case. This will provide a new proof of the $q$-Gauss summation formula. We shall work with the Askey-Wilson operator which arises from the geometric canonical form

$$
\mathrm{D} f(z)=\frac{f\left(q^{1 / 2} z\right)-f\left(q^{-1 / 2} z\right)}{q^{1 / 2} z-q^{-1 / 2} z} .
$$

Given $\alpha, \beta \in \mathbb{C}$ and $|q|<1$, we consider the function

$$
f(x)=\frac{(\alpha x ; q)_{\infty}}{(\beta x ; q)_{\infty}}=\prod_{j=0}^{\infty} \frac{1-q^{j} \alpha x}{1-q^{j} \beta x} .
$$

By induction, it is not difficult to check that

$$
\partial_{k} f(z)=\left(\prod_{j=0}^{k-1} \frac{\beta-q^{j} \alpha}{1-q^{j+1}}\right) \frac{\left(\alpha z q^{k / 2} ; q\right)_{\infty}}{\left(\beta z q^{-k / 2} ; q\right)_{\infty}} .
$$

Therefore, taking $\xi \in \mathbb{C}$ and $z_{t}=q^{t} \xi$ in Theorem 2.6, we obtain

$$
\frac{(\alpha x ; q)_{\infty}(\beta \xi ; q)_{\infty}}{(\beta x ; q)_{\infty}(\alpha \xi ; q)_{\infty}}=\sum_{k=0}^{\infty} \frac{(\xi / x ; q)_{k}}{(q ; q)_{k}(\alpha \xi ; q)_{k}}\left(\prod_{j=0}^{k-1}\left(\beta-q^{j} \alpha\right)\right) x^{k} .
$$

In particular, with the usual notation for basic hypergeometric series,

$$
{ }_{2} \phi_{1}\left[\begin{array}{c}
\xi / x, \alpha / \beta \\
\alpha \xi
\end{array} ; q, \beta x\right]=\frac{(\alpha x, \beta \xi ; q)_{\infty}}{(\beta x, \alpha \xi ; q)_{\infty}} .
$$

In other words, we have obtained the $q$-Gauss classical summation formula.

3.2. An estimate for $q$-shifted factorials. Here we provide an estimate for $q$ shifted factorials that will be needed below. Although we just need a weaker estimate, we have included the sharpest result we know since it might be of independent interest.

Lemma 3.1. The following assertions hold for $0<q<1$ :

(a) There exists some $\mathrm{C}_{q}>0$ such that every $x \in \mathbb{C}$ satisfies

$$
\left|\prod_{j=0}^{\infty}\left(1-q^{j} x\right)\right| \leq \mathrm{C}_{q}|x|^{\frac{1}{2}+\frac{\log |x|}{2 \log q^{-1}}} .
$$

(b) Let $\mathcal{A}$ be any closed set in $\mathbb{C}$ with $q \mathcal{A} \subset \mathcal{A}$ and $q^{-j} \notin \mathcal{A}$ for $j=0,1,2, \ldots$ Then, given any $\delta>0$ there exists a constant $\mathrm{C}_{q}(\mathcal{A}, \delta)>0$ such that for all $x \in \mathcal{A} \backslash \mathbb{D}_{\delta}$, we have

$$
\left|\prod_{j=0}^{\infty}\left(1-q^{j} x\right)\right| \geq \mathrm{C}_{q}(\mathcal{A}, \delta)|x|^{\frac{1}{2}+\frac{\log |x|}{2 \log q^{-1}}} .
$$

Proof. Let us write

$$
h(x)=\prod_{j=0}^{\infty}\left(1-q^{j} x\right) \quad \text { and } \quad h_{n}(x)=\prod_{j=0}^{n-1}\left(1-q^{j} x\right) .
$$


We begin by proving the upper estimate. Since the function $|x|^{\frac{1}{2}+\frac{\log |x|}{2 \log q^{-1}}}$ blows up at $x=0$, we may assume that $|x|>1$. Then we fix the only integer $m$ satisfying the estimates $q^{m}|x| \leq 1<q^{m-1}|x|$ and observe that

$$
|h(x)|=\left|h\left(q^{m} x\right) \prod_{j=0}^{m-1}\left(1-q^{j} x\right)\right|=|x|^{m} q^{\left(\begin{array}{c}
m \\
2
\end{array}\right)}\left|h(a) h_{m}(b)\right|,
$$

where $a=q^{m} x$ and $b=q^{-m+1} x^{-1}$. Our choice of $m$ implies

$$
m=\frac{\log |x|}{\log q^{-1}}+\rho \quad \text { for some } \quad 0 \leq \rho<1 .
$$

Moreover, a simple computation gives

$$
|x|^{m} q^{\left(\begin{array}{c}
m \\
2
\end{array}\right)}=q^{\frac{\rho(\rho-1)}{2}}|x|^{\frac{1}{2}+\frac{\log |x|}{2 \log q^{-1}}} .
$$

Therefore, since $|a| \leq 1$ and $q \leq|b|<1$, we have $\left|h(a) h_{n}(b)\right| \leq h(-1)^{2}$ and we deduce the assertion with constant $\mathrm{C}_{q}=q^{-1 / 8} h(-1)^{2}$. Now we prove the lower estimate. Let us consider the set

$$
\mathcal{B}=\left\{z \in \mathbb{C}: z^{-1} \in \mathcal{A}, q \leq|z| \leq 1\right\} .
$$

The function $h$ does not vanish on $\mathcal{A} \cup \mathcal{B}$. Therefore, since $h_{n} \rightarrow h$ uniformly over compact sets, we deduce that $\min \left\{\left|h_{n}(z)\right|,|h(z)|\right\} \geq \mathrm{C}_{q}(\mathcal{A})$ for some $\mathrm{C}_{q}(\mathcal{A})$, for all $n \geq 1$ and all $z \in \mathcal{A} \cup \mathcal{B}$ with $|z| \leq 1$. Thus, if $|x|>1$ we deduce from (3) and (44) (recall that $a \in \mathcal{A}$ and $b \in \mathcal{B}$ ) that

$$
\left|\prod_{j=0}^{\infty}\left(1-q^{j} x\right)\right| \geq \mathrm{C}_{q}(\mathcal{A})|x|^{\frac{1}{2}+\frac{\log |x|}{2 \log q^{-1}}} \quad \text { for all } x \in \mathcal{A} \backslash \overline{\mathbb{D}}_{1} .
$$

The assertion of $x \in \mathcal{A}$ with $\delta \leq|x| \leq 1$ follows by replacing $\mathrm{C}_{q}(\mathcal{A})$ by $\mathrm{C}_{q}(\mathcal{A}, \delta)$.

Remark 3.2. Let us observe how to construct sets of type $\mathcal{A}$. Since $q^{-j} \notin \mathcal{A}$ for $j=0,1,2, \ldots$ and $\mathcal{A}$ is closed, there must exist an open neighborhood $\mathcal{U}$ of 1 such that $\mathcal{A} \cap \mathcal{U}=\emptyset$. Now, recalling that $q \mathcal{A} \subset \mathcal{A}$ if and only if $q^{-1} \mathcal{A}^{c} \subset \mathcal{A}^{c}$, we must have that the neighborhood of $q^{-j}$ defined by $q^{-j} \mathcal{U}$ is contained in $\mathcal{A}^{c}$. Thus, the biggest $\mathcal{A}$ 's are sets generated by a neighborhood $\mathcal{U}$ of 1 in the following way:

$$
\mathcal{A}=\bigcap_{j=0}^{\infty}\left(q^{-j} \mathcal{U}\right)^{c}
$$

3.3. Trigonometric case. Now we apply Theorem 2.7 to

$$
f_{\alpha \beta}(x)=f_{\alpha \beta}\left(\frac{u+u^{-1}}{2}\right)=\frac{(\alpha u ; q)_{\infty}(\alpha / u ; q)_{\infty}}{(\beta u ; q)_{\infty}(\beta / u ; q)_{\infty}} \quad \text { with } \quad x=\frac{1}{2}\left(u+u^{-1}\right)
$$

and $(\alpha, \beta) \in \mathbb{R} \times \mathbb{R}_{+}$. As a function of $x, f_{\alpha, \beta}$ is well-defined since $(\gamma u, \gamma / u ; q)_{\infty}$ is symmetric under the mapping $u \mapsto u^{-1}$. Moreover, it is analytic in a neighborhood of the left half-plane (as a function of $x$ ) since $(\gamma u, \gamma / u ; q)_{\infty}$ is analytic in $\mathbb{C} \backslash\{0\}$ for any $\gamma \in \mathbb{C}$ and has zeros in $\mathbb{R}_{+}$when $\gamma \in \mathbb{R}_{+}$. We shall use the Askey-Wilson operator which arises from the trigonometric canonical form

$$
\mathrm{D} f\left(\frac{u+u^{-1}}{2}\right)=\frac{2}{\left(\lambda-\lambda^{-1}\right)\left(u-u^{-1}\right)}\left[f\left(\frac{\lambda u+\lambda^{-1} u^{-1}}{2}\right)-f\left(\frac{\lambda^{-1} u+\lambda u^{-1}}{2}\right)\right]
$$


with $q=\lambda^{2}$ and $0<\lambda<1$. Grouping the common factors, it can be checked that

$$
\mathrm{D} f_{\alpha \beta}\left(\frac{u+u^{-1}}{2}\right)=2 \frac{\alpha-\beta}{q-1} f_{\lambda \alpha, \lambda^{-1} \beta}\left(\frac{u+u^{-1}}{2}\right) \text {. }
$$

Moreover, by induction on $k$ we obtain

$$
\partial_{k} f_{\alpha \beta}\left(\frac{u+u^{-1}}{2}\right)=\frac{2^{k}}{(q ; q)_{k}} \prod_{j=0}^{k-1}\left(\beta-q^{j} \alpha\right) f_{\lambda^{k} \alpha, \lambda^{-k} \beta}\left(\frac{u+u^{-1}}{2}\right) .
$$

In particular, taking $z_{t}=\frac{1}{2}\left(q^{t} \xi+q^{-t} \xi^{-1}\right)$ with $\xi<0$, we can write

$$
\partial_{k} f_{\alpha \beta}\left(z_{k / 2}\right)=\frac{(2 \beta)^{k}(\alpha / \beta ; q)_{k}}{\left(q, \alpha \xi, \beta / q^{k} \xi ; q\right)_{k}} f_{\alpha \beta}\left(\frac{\xi+\xi^{-1}}{2}\right) \text {. }
$$

According to Theorem 2.3 and

$$
\frac{\zeta+\zeta^{-1}}{2}-\frac{\eta+\eta^{-1}}{2}=\frac{\left((\zeta \eta)^{1 / 2}-(\zeta \eta)^{-1 / 2}\right)\left((\zeta / \eta)^{1 / 2}-(\zeta / \eta)^{-1 / 2}\right)}{2}
$$

the Taylor polynomials of $f_{\alpha, \beta}$ are

$$
\mathcal{S}_{n} f_{\alpha \beta}\left(\frac{u+u^{-1}}{2}\right)=f_{\alpha \beta}\left(\frac{\xi+\xi^{-1}}{2}\right) \sum_{k=0}^{n} \frac{(\alpha / \beta, \xi u, \xi / u ; q)_{k}}{(q, \alpha \xi, q \xi / \beta ; q)_{k}} q^{k}
$$

In other words, in terms of Theorem 2.7 we have

$$
\mathcal{S}_{\infty} f_{\alpha \beta}\left(\frac{u+u^{-1}}{2}\right)=\frac{(\alpha \xi, \alpha / \xi ; q)_{\infty}}{(\beta \xi, \beta / \xi ; q)_{\infty}}{ }_{3} \phi_{2}\left[\begin{array}{c}
\alpha / \beta, \xi u, \xi / u \\
\alpha \xi, q \xi / \beta
\end{array} ; q, q\right] .
$$

On the other hand, the subsequence $z_{0}, z_{1}, z_{2}, \ldots$ lies in $\mathbb{R}_{-}$with $\sum_{k \geq 0} 1 /\left|z_{k}\right|<\infty$. Thus, in order to apply Theorem 2.7, it suffices to check whether the bound (2) holds for some $M>0$. To that aim we shall assume that $|u| \geq 1$ and

$$
|x| \geq 2 \max \left\{|\alpha|,|\alpha|^{-1}, \beta, \beta^{-1}\right\} \text {. }
$$

This implies $\frac{1}{4}|u| \leq|x| \leq|u|$ so that $|\alpha / u|, \beta /|u| \leq 1 / 2$ and the functions $(\alpha / u ; q)_{\infty}$, $(\beta / u ; q)_{\infty}$ are bounded from above and below. Moreover, according to Lemma 3.1. we obtain the following estimate:

$$
\left|(\alpha u ; q)_{\infty}\right| \leq \mathrm{C}_{q}(\alpha)|u|^{\frac{1}{2}+\frac{\log |u|}{2 \log q^{-1}}}|\alpha|^{\frac{\log |u|}{2 \log q^{-1}}}|u|^{\frac{\log |\alpha|}{2 \log q^{-1}}}=\mathrm{C}_{q}(\alpha)|u|^{\frac{1}{2}+\frac{\log |u|}{2 \log q^{-1}}+\frac{\log |\alpha|}{\log q^{-1}}}
$$

with $\mathrm{C}_{q}(\alpha)=\mathrm{C}_{q}|\alpha|^{\frac{1}{2}+\frac{\log |\alpha|}{2 \log q^{-1}}}$. Similarly, when $\operatorname{Re} u \leq 0$ (equivalently $\operatorname{Re} x \leq 0$ ),

$$
\left|(\beta u ; q)_{\infty}\right| \geq \mathrm{C}_{q}(\beta)|u|^{\frac{1}{2}+\frac{\log |u|}{2 \log q^{-1}}}|\beta|^{\frac{\log |u|}{2 \log q^{-1}}}|u|^{\frac{\log |\beta|}{2 \log q^{-1}}}=\mathrm{C}_{q}(\beta)|u|^{\frac{1}{2}+\frac{\log |u|}{2 \log q^{-1}}+\frac{\log |\beta|}{\log q^{-1}}} .
$$

In summary, we have seen that

$$
\left|f_{\alpha, \beta}(x)\right| \leq \mathrm{C}_{q}(\alpha, \beta)|u|^{\mathrm{M}} \leq 4 \mathrm{C}_{q}(\alpha, \beta)(1+|x|)^{\mathrm{M}} \quad \text { with } \quad \mathrm{M}=\frac{\log |\alpha / \beta|}{\log q^{-1}},
$$

whenever $\operatorname{Re} x \leq 0$ and $|x| \geq 2 \max \left(|\alpha|,|\alpha|^{-1}, \beta, \beta^{-1}\right)$. However, the last restriction on $x$ can be dropped by continuity and we are in the hypotheses of Theorem 2.7 This gives the identity $f_{\alpha, \beta}(x)=\mathcal{S}_{\infty} f_{\alpha, \beta}(x)+\mathcal{R}_{\infty} f_{\alpha, \beta}(x)$ for $\operatorname{Re} x<0$. Letting $f_{\gamma}(x)=(\gamma u, \gamma / u ; q)_{\infty}\left(\right.$ so that $\left.f_{\alpha, \beta}=f_{\alpha} / f_{\beta}\right)$, we have

$$
\mathrm{H}(x)=\prod_{j=0}^{\infty}\left(1-\frac{u+u^{-1}}{q^{j} \xi+q^{-j} \xi^{-1}}\right)=\prod_{j=0}^{\infty} \frac{\left(1-q^{j} \xi u\right)\left(1-q^{j} \xi / u\right)}{1+q^{2 j} \xi^{2}}=\left(-\xi^{2} ; q^{2}\right)_{\infty}^{-1} f_{\xi}(x) .
$$


Therefore, we have

$$
\mathcal{R}_{\infty} f_{\alpha, \beta}(x)=\frac{1}{2 \pi i} \int_{-i \infty}^{i \infty} \frac{f_{\alpha, \beta}(y)}{y-x} \frac{\mathrm{H}(x)}{\mathrm{H}(y)} d y=\frac{1}{2 \pi i} \int_{-i \infty}^{i \infty} \frac{f_{\alpha}(y) f_{\xi}(x)}{f_{\beta}(y) f_{\xi}(y)} \frac{d y}{y-x} .
$$

Taking $y=\frac{1}{2}\left(v+v^{-1}\right)$, it follows from (6) and (7) that

(8) $\frac{(\alpha u, \alpha / u ; q)_{\infty}}{(\beta u, \beta / u ; q)_{\infty}}=\frac{(\alpha \xi, \alpha / \xi ; q)_{\infty}}{(\beta \xi, \beta / \xi ; q)_{\infty}}{ }_{3} \phi_{2}\left[\begin{array}{c}\alpha / \beta, \xi u, \xi / u \\ \alpha \xi, q \xi / \beta\end{array} ; q, q\right]$

$$
+\frac{1}{2 \pi i} \int_{-i \infty}^{i \infty} \frac{(\alpha v, \alpha / v, \xi u, \xi / u ; q)_{\infty}}{(\beta v, \beta / v, \xi v, \xi / v ; q)_{\infty}} \frac{\left(1-v^{2}\right) u}{(u-v)(u v-1) v} d v
$$

for $(\alpha, \beta) \in \mathbb{R} \times \mathbb{R}_{+}, \xi<0$ and $\operatorname{Re} u<0$. This summation formula is new as far as we know. It can be regarded as a non-symmetrized version of the non-terminating $q$-Saalschütz sum. To see this, we observe that an obvious reformulation of Theorem 2.7 gives (8) when $\operatorname{Re} x=0$ with $\mathcal{R}_{\infty} f_{\alpha, \beta}(x)$ replaced by

$$
\mathcal{R}_{\infty}(x ; \alpha, \beta, \xi)=\frac{1}{2 \pi i}\left(\int_{-i \infty}^{x-\delta i}+\int_{\gamma_{x}^{+}}+\int_{x+\delta i}^{i \infty}\right) \frac{f_{\alpha}(y) f_{\xi}(x)}{f_{\beta}(y) f_{\xi}(y)} \frac{d y}{y-x},
$$

with $\gamma_{x}^{+}(t)=x-i \delta e^{i t}$ for $0 \leq t \leq \pi$. Transposing $(\beta, \xi)$ and changing signs,

$$
\begin{aligned}
\mathcal{R}_{\infty}(-x ;-\alpha,-\xi,-\beta) & =\frac{1}{2 \pi i}\left(\int_{-i \infty}^{-x-\delta i}+\int_{\gamma_{-x}^{+}}+\int_{-x+\delta i}^{i \infty}\right) \frac{f_{\alpha}(-y) f_{\beta}(x)}{f_{\xi}(-y) f_{\beta}(-y)} \frac{d y}{y+x} \\
& =\frac{1}{2 \pi i}\left(\int_{i \infty}^{x+\delta i}+\int_{\gamma_{\bar{x}}}+\int_{x-\delta i}^{-i \infty}\right) \frac{f_{\alpha}(y) f_{\beta}(x)}{f_{\beta}(y) f_{\xi}(y)} \frac{d y}{y-x},
\end{aligned}
$$

where $\gamma_{x}^{-}(t)=x+i \delta e^{i t}$ for $0 \leq t \leq \pi$. Calculating a residue we conclude

$$
\frac{f_{\alpha}(x)}{f_{\beta}(x) f_{\gamma}(x)}=\frac{\mathcal{R}_{\infty}(x ; \alpha, \beta, \xi)}{f_{\xi}(x)}+\frac{\mathcal{R}_{\infty}(-x ;-\alpha,-\xi,-\beta)}{f_{\beta}(x)} .
$$

The non-terminating $q$-Saalschütz sum follows easily from (8) and (9) in the form

$$
\begin{aligned}
\frac{(\alpha u, \alpha / u ; q)_{\infty}}{(\beta u, \beta / u, \xi u, \xi / u ; q)_{\infty}} & =\frac{(\alpha \xi, \alpha / \xi ; q)_{\infty}}{(\beta \xi, \beta / \xi, \xi u, \xi / u ; q)_{\infty}}{ }_{3} \phi_{2}\left[\begin{array}{c}
\alpha / \beta, \xi u, \xi / u \\
\alpha \xi, q \xi / \beta
\end{array} ; q, q\right] \\
& +\frac{(\alpha \beta, \alpha / \beta ; q)_{\infty}}{(\beta u, \beta / u, \xi \beta, \xi / \beta ; q)_{\infty}}{ }_{3} \phi_{2}\left[\begin{array}{c}
\alpha / \xi, \beta u, \beta / u \\
\alpha \beta, q \beta / \xi
\end{array} ; q, q\right] .
\end{aligned}
$$

Note that our restrictions on $\alpha, \beta$ and $\xi$ disappear after analytic extension.

Remark 3.3. Going back to the example considered in Paragraph 3.1, we may consider the P-sequence $z_{t}=q^{-t} \xi$ and apply Theorem 2.7 instead of Theorem 2.6. In that case, similar arguments give rise to a non-symmetrized version of the non-terminating $q$-Vandermonde sum

$$
\frac{(\alpha x ; q)_{\infty}}{(\beta x ; q)_{\infty}}=\frac{(\alpha \xi ; q)_{\infty}}{(\beta \xi ; q)_{\infty}}{ }_{2} \phi_{1}\left[\begin{array}{c}
\alpha / \beta, x / \xi \\
q / \beta \xi,
\end{array} ; q, q\right]+\frac{1}{2 \pi i} \int_{-i \infty}^{i \infty} \frac{(\alpha y, x / \xi ; q)_{\infty}}{(\beta y, y / \xi ; q)_{\infty}} \frac{d y}{y-x}
$$

\section{Binomial theorem}

As announced in the Introduction, we finish with a list of binomial type formulas adapted to our classification into canonical forms. We must recall that the same techniques we are using here were already employed by Ismail in 2 to obtain the binomial type sum for the trigonometric canonical form; see below. Let $f \in \mathbb{P}_{n}[x]$ and let $\left(z_{t}\right)$ be a $\mathrm{P}$-sequence with $z_{0}, z_{1}, \ldots, z_{n}$ pairwise distinct. Then, it follows 
from Remark 2.5 that $\mathcal{R}_{m} f=0$ for any $m \geq n$. Moreover, by a simple continuity argument, we may drop the assumption that $z_{0}, z_{1}, \ldots, z_{n}$ are pairwise distinct. We need to use the $q$-binomial coefficients

$$
\left[\begin{array}{l}
r \\
k
\end{array}\right]_{q}=\frac{(q ; q)_{r}}{(q ; q)_{k}(q ; q)_{r-k}}, \quad \text { where }(x ; q)_{n}=\prod_{k=0}^{n-1}\left(1-q^{k} x\right) .
$$

Theorem 4.1. Given two P-sequences $\left(y_{t}\right)$ and $\left(z_{t}\right)$, we have

$$
\prod_{k=0}^{n-1}\left(x-z_{k}\right)=\sum_{k=0}^{n}\left[\begin{array}{l}
n \\
k
\end{array}\right]_{q} q^{-k(n-k) / 2} \prod_{j=0}^{k-1}\left(x-y_{j}\right) \prod_{j=0}^{n-k-1}\left(y_{k / 2}-z_{j+\frac{k}{2}}\right) .
$$

Proof. We claim that the following identity holds:

$$
\partial_{k} \Phi_{n}(x, \cdot)=\left(\prod_{j=0}^{k-1} \frac{\lambda^{n-j}-\lambda^{j-n}}{\lambda^{k-j}-\lambda^{j-k}}\right) \Phi_{n-k}(x, \cdot), \quad 0 \leq k \leq n .
$$

This can be checked by induction on $k$ and applying the last identity in the proof of Lemma 2.2. In particular, since $\Phi_{n}\left(z_{(n-1) / 2}, \cdot\right) \in \mathbb{P}_{n}[x]$, Theorem 2.3 gives

$$
\Phi_{n}\left(z_{(n-1) / 2}, x\right)=\sum_{k=0}^{n}\left(\prod_{j=0}^{k-1} \frac{\lambda^{n-j}-\lambda^{j-n}}{\lambda^{k-j}-\lambda^{j-k}}\right) \Phi_{n-k}\left(z_{(n-1) / 2}, y_{k / 2}\right) \prod_{j=0}^{k-1}\left(x-y_{j}\right) .
$$

By the definition of $\Phi_{k}$ and the $q$-binomial coefficient, the proof is completed.

Theorem 4.1 is symmetric under the transformation $q \mapsto q^{-1}$. Moreover, when $q=1$ we must take the obvious limits. Clearly, for the continuous canonical form, Theorem 4.1 is nothing but Newton's binomial theorem. Other explicit formulas of binomial type arise from the remaining canonical forms. Moreover, they can be rewritten in terms of the basic hypergeometric function. In Table II we summarize the binomial type expressions and we mention the corresponding hypergeometric

\begin{tabular}{|c|c|c|}
\hline C & $(x-z)^{n}=\sum_{k=0}^{n}\left(\begin{array}{l}n \\
k\end{array}\right)(x-y)^{k}(y-z)^{n-k}$ & Newton \\
\hline A & $\left(\begin{array}{c}x-z \\
n\end{array}\right)=\sum_{k=0}^{n}\left(\begin{array}{c}x-y \\
k\end{array}\right)\left(\begin{array}{l}y-z \\
n-k\end{array}\right)$ & Gauss $^{\dagger}$ \\
\hline $\mathbf{Q}$ & $(u+w)_{n}\left(\begin{array}{c}u-w \\
n\end{array}\right)=\sum_{k=0}^{n}\left(\begin{array}{c}u-v \\
k\end{array}\right)(u+v)_{k}\left(\begin{array}{c}v-w \\
n-k\end{array}\right)(v+w+k)_{n-k}$ & Pfaff \\
\hline G & $\prod_{k=0}^{n-1}\left(x-q^{k} z\right)=\sum_{k=0}^{n}\left[\begin{array}{l}n \\
k\end{array}\right]_{q} \prod_{j=0}^{k-1}\left(x-q^{j} y\right) \prod_{j=0}^{n-k-1}\left(y-q^{j} z\right)$ & $q-$ Gauss $^{\dagger}$ \\
\hline $\mathbf{T}$ & $\frac{(w u, w / u ; q)_{n}}{(q ; q)_{n}}=\sum_{k=0}^{n} \frac{(v u, v / u ; q)_{k}}{(q ; q)_{k}} \frac{\left(w v q^{k}, w / v ; q\right)_{n-k}}{(q ; q)_{n-k}}\left(\frac{w}{v}\right)^{k}$ & q-Saalschütz \\
\hline
\end{tabular}
formula.

TABLE II. Binomial type summation formulas

\section{Binomial form Sum}

The reader is referred to Gasper and Rahman's book [1] to see these summation formulas. The symbol $\dagger$ means that, if we rewrite the binomial type formula in 
terms of hypergeometric summation formulas, what we obtain is a terminating form of the corresponding non-terminating summation formula. Although we leave the details for the interested reader, we should point out the changes of variables employed in the quadratic and trigonometric canonical forms:

$$
\begin{aligned}
& \text { (Q) } x=u^{2} \quad y_{k}=(v+k)^{2} \quad z_{k}=(w+k)^{2} \\
& \text { (T) } \quad x=\frac{1}{2}\left(u+u^{-1}\right) \quad y_{k}=\frac{1}{2}\left(q^{k} v+q^{-k} v^{-1}\right) \quad z_{k}=\frac{1}{2}\left(q^{k} w+q^{-k} w^{-1}\right) \text {. }
\end{aligned}
$$

In the trigonometric case, we also need to use the factorization formula (5).

\section{ACKNOWLEDGMENTS}

The authors wish to thank the referee for several comments that gave rise to a significant improvement of this paper. This research was supported in part by the Project MTM2004-00678, Spain.

\section{REFERENCES}

1. G. Gasper and M. Rahman, Basic hypergeometric series. With a foreword by Richard Askey, Encyclopedia of Mathematics and its Applications 35, Cambridge Univ. Press, 1990. MR 1052153 (91d:33034)

2. M.E.H. Ismail, The Askey-Wilson operator and summation theorems, M. Ismail, M.Z. Nashed, A. Zayed, A. Ghaleb (Eds.), Mathematical Analysis, Wavelets and Signal Processing, Contemp. Math. 190 (1995), 171-178. MR.1354852 (96j:33011)

3. M.E.H. Ismail and D. Stanton, q-Taylor theorems, polynomial expansions, and interpolation of entire functions, J. Approx. Th. 123 (2003), 125-146. MR.1985020 (2004g:30040)

4. M.E.H. Ismail and D. Stanton, Applications of q-Taylor theorems, J. Comp. Appl. Math. 153 (2003), 259-272. MR 1985698 (2004f:33035)

5. J.M. Marco and J. Parcet, A new approach to the theory of classical hypergeometric polynomials. Trans. Amer. Math. Soc. 358 (2006), 183-214. MR2171229

6. R.V. Wallisser, On entire functions assuming integer values in a geometric sequence, Théorie des nombres (Quebec, PQ, 1987), 981-989, de Gruyter, Berlin, 1989. MR1024616 (90j:11067)

Department of Mathematics, Universidad Autónoma de Madrid, 28049, Madrid, Spain

E-mail address: bernardo.lopez@uam.es

Department of Mathematics, Universidad Autónoma de Madrid, 28049, Madrid, Spain

Centre de Recerca Matemàtica, Universidad Autónoma de Barcelona, Apartat 50, 08193, Bellaterra, Barcelona, Spain

E-mail address: jparcet@crm.es 